\title{
Multifractal properties of Pyrex and silicon surfaces blasted with sharp particles
}

\author{
Z. Moktadir ${ }^{\mathrm{a}, *}$, M. Kraft ${ }^{\mathrm{a}}$, H. Wensink ${ }^{\mathrm{b}}$ \\ ${ }^{a}$ School of Electronics and Computer Science, Southampton University, Southampton, SO17 1BJ, United Kingdom \\ ${ }^{\mathrm{b}}$ Transducers Science and Technology, MESA + Research Institute, University of Twente, The Netherlands
}

Received 23 July 2007; received in revised form 3 October 2007

Available online 22 November 2007

\begin{abstract}
The blasting of brittle materials with sharp particles is an important fabrication technology in many industrial processes. In particular, for microsystems, it allows the production of devices with feature sizes down to few tens of microns. An important parameter of this process is the surface roughness of post-blasted surfaces. In this work the scaling properties of Pyrex glass and silicon surfaces after bombardment with alumina particles are investigated. The targets were bombarded at normal incidence using alumina particles with two different average sizes, $29 \mu \mathrm{m}$ and $9 \mu \mathrm{m}$. This investigation indicates that the resulting surfaces are multifractal. Applying multifractal detrended fluctuation analysis (MFDFA) allowed us to determine the singularity spectrum of the surfaces. This spectrum did not depend on the target material or on the size of the particles. Several parameters quantifying relevant quantities were determined. It was found that long range correlations are responsible for the observed multifractal behaviour.
\end{abstract}

(c) 2007 Elsevier B.V. All rights reserved.

Keywords: Pyrex; Silicon; Sharp particles; Multifractals; Singularity spectrum

\section{Introduction}

Powder blasting technology is among several techniques used in the micromachining [1-3] of devices on silicon and other materials. With such abrasive techniques, one can achieve high erosion rates; higher than those that can be obtained with conventional dry or wet etching processes such as plasma etching and chemical etching. In the field of microelectromechanical systems (MEMS), powder blasting has already been used for the fabrication of inertial sensors [2], peristaltic micropumps [3] and miniaturized capillary electrophoresis chips [4]. Erosion with sharp particles is also a widely used technique in aerospace and automotive industries. Because of its involvement in many applications, it is important to investigate the surface morphology of blasted surfaces resulting from erosion since the performance of many devices will depend on the surface roughness. Such investigation will also give an insight into the physical mechanisms at work during the bombardment of materials with particles. The mechanisms involved in the erosion of brittle materials with sharp particles have been the subject of several studies. Several models have been developed [5,6] that are based on simple static indentation theory. These models empirically relate the

\footnotetext{
* Corresponding author. Tel.: +44 2380593126.

E-mail address: zm@ecs.soton.ac.uk (Z. Moktadir).
} 


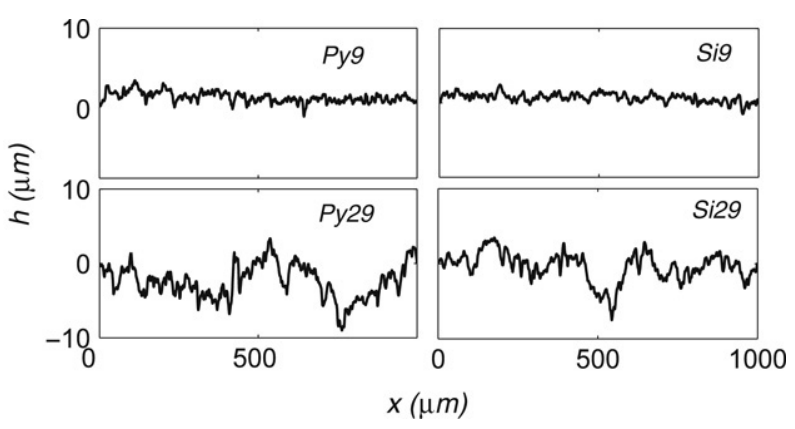

Fig. 1. Surface profiles after blasting of Pyrex and silicon with particles of size $\delta=9 \mu \mathrm{m}$ (Py9 and Si9), and with particles of size $\delta=29 \mu \mathrm{m}$ (Py29 and Si29).

erosion rate to the material's properties such as the fracture toughness, the hardness and the Young modulus of the material. These models simply state that an indentation force is generated by the impact of bombarding particles which, in turn, results in the formation of crack patterns. Some cracks penetrate the material radially away from the surface into the bulk material; others will nucleate and form a lateral ring parallel to the surface [7-9]. The radial cracking process was extensively studied $[10,11]$ and was used as a method for materials toughness measurement [12]. Lateral cracks are responsible for the removal of material in abrasive and wear experiments on brittle materials [13-15].

In separate studies, substantial work was dedicated to the understanding of fracture surfaces [16] in brittle materials. These studies focus on the scaling properties of fracture surfaces resulting from an applied load on the material. These surfaces are found to be self-affine, i.e. the root mean square surface fluctuations (defined as $\sigma^{2}=1 / N \sum_{i=1}^{N}(h(i)-\bar{h})$, where $h(i)$ is the surface profile at a point $i, \bar{h}$ is its average value and $N$ the number of data points in the profile) averaged over a distance $L$ follows the scaling relation [17]

$$
\sigma \sim L^{\alpha}
$$

where the scaling exponent $\alpha$ is often called the roughness or the Hurst exponent. In a variety of materials, the value of $\alpha$ was found to be approximately 0.8 over two or three decades of scaling range. For this reason, it was conjectured to be universal, i.e. independent of the material, the fracture mode and the fracture toughness $[16,18,19]$.

The present work is dedicated to the study of the scaling properties of surfaces resulting from a bombardment by sharp particles. Pyrex glass (borosilicate glass) and silicon were the materials used in this investigation. We will investigate the effect of the material and the size of the bombarding particles on those properties.

\section{Experiments}

In the experiments carried out, surfaces are exposed to a directed particle jet, which results in mechanical material removal. The particles are accelerated towards the target with a high pressure air flow through a circular nozzle (with a diameter of $1.5 \mu \mathrm{m}$ ). The particles hit the target under normal incidence, with an average speed of $290 \mathrm{~m} / \mathrm{s}$, in a ventilated box. A lateral movement of the target ensures an evenly etched surface. The average diameters of the bombarding alumina particles were $9 \mu \mathrm{m}$ and $29 \mu \mathrm{m}$. We performed measurements on one-dimensional cuts of blasted surfaces using a mechanical surface profiler (Sloan Dektak II), over a length of $1 \mathrm{~mm}$. Each scanned profile is made of 8000 data points. The vertical resolution of the profiler was $10 \AA$. Typical profiles obtained after blasting Pyrex and silicon surfaces with alumina particles are shown in Fig. 1. Note the difference in roughness amplitude between the two cases corresponding to different particle sizes. Large amplitudes are obtained when the targets are blasted with large particles. The typical rms surface roughness is $\sigma=0.7 \mu \mathrm{m}$ and $\sigma=0.65 \mu \mathrm{m}$ for Pyrex and silicon respectively, when $9 \mu \mathrm{m}$ particles are used. In Table 1, measured values of the surface rms roughness are shown, as a function of the bombarding particle sizes and the target material. Samples bombarded with particles having a diameter $\delta$ are denoted as $X \delta$, where $X$ denotes the material's symbol. For example Si29 means silicon bombarded with particles having a diameter of $29 \mu \mathrm{m}$. 
Table 1

Values of the rms roughness as a function of the particles size and the target material

\begin{tabular}{llr}
\hline Particles size & $9 \mu \mathrm{m}$ & $29 \mu \mathrm{m}$ \\
Pyrex & $0.7 \pm 0.02 \mu \mathrm{m}$ & $2.2 \pm 0.02 \mu \mathrm{m}$ \\
Silicon & $0.65 \pm 0.01 \mu \mathrm{m}$ & $2.3 \pm 0.01 \mu \mathrm{m}$ \\
\hline
\end{tabular}

\section{Multifractal properties and the singularity spectrum}

Rough surfaces exhibiting a range of roughness exponents are called multifractal [20]. Our aim is to determine the full range of local roughness exponents for the blasted surfaces using multifractal analysis. To do so, we characterized the profiles by computing the so-called singularity spectrum, which determines the distribution of the whole range of local roughness exponents. We used the multifractal detrended fluctuation analysis (MFDFA) [21]. This method has become popular thanks to its simplicity and its easy computer implementation. Other methods exist such as the wavelet transform modulus maxima method (WTMM) [22] but the MFDFA has several advantages [23]. The MFDFA is the extension of the detrended fluctuation (DFA) [24] method which was used to compute the roughness exponent of monofractal signals and for the identification of long range correlations in non-stationary time series [21]. MFDFA is an efficient tool for eliminating undesirable trends in fluctuations. This method applied to our experimental data can be summarized as follows: Given a profile $h(i), i=1, \ldots, N$, we compute the integrated profile,

$$
H(i)=\sum_{j=1}^{i}(h(j)-\langle h\rangle)
$$

where $N$ is the number of data points and $\langle h\rangle$ is the mean height of the profile. The whole profile is subdivided into $M_{L}=N / L$ non-overlapping segments of length $L$ (here $L$ is the number of data points in each segment). Since $M_{L}$ is not always an integer, some data will be ignored during this procedure. To take them into account, the subdivision is performed from both ends of the profile, which results in $2 M_{L}$ segments. In each segment $n$ the polynomial trend $P_{n}$ is subtracted from the data. This polynomial is determined by a least squares fit to the data in each segment. Polynomials of degrees higher than 1 can be used, corresponding to MFDFA2, MFDFA3, etc. After detrending in each segment, the variance of the result is calculated:

$$
F^{2}(n, L)=\frac{1}{L} \sum_{j=1}^{j=L}\left[H((n-1) L+j)-P_{n}(j)\right]^{2}
$$

This expression is then averaged over all segments $n$ and the value of the $q$ th-order fluctuation function is calculated:

$$
F_{q}(L)=\left(\frac{1}{2 M_{L}} \sum_{n=1}^{2 M_{L}} F^{2}(n, L)^{q / 2}\right)^{1 / q} .
$$

Here $q$ is a real number. For a fractal profile, $F_{q}(L)$ follows a power law relation at large scales, i.e.

$$
F_{q}(L) \sim L^{h(q)} .
$$

The exponent $h(q)$ is called the "generalized Hurst exponent". For a monofractal profile, $h(q)=$ const, while for a multifractal profile, $h$ is a function of $q$. For positive values of $q, h(q)$ describes the scaling behaviour of the segments with large fluctuations, while for negative values of $q, h(q)$ describes the scaling behaviour of the segments with small fluctuations. The singularity spectrum $f(\alpha)$ is calculated by obtaining the Legendre transform [25] of $(q, \tau(q))$ with $\tau(q)=q h(q)-1$, resulting in

$$
\begin{aligned}
& \alpha=\frac{\mathrm{d} \tau(q)}{\mathrm{d} q} \\
& f(\alpha)=q \alpha-\tau(q) .
\end{aligned}
$$

The spectrum $f(\alpha)$ can be interpreted as the fractal dimension of a subset of points in the profile characterized by the singularity strength $\alpha$ (the local roughness exponent). For a monofractal profile, $\alpha=\alpha_{0}$ and $f(\alpha)=1$, where $\alpha_{0}$ is 


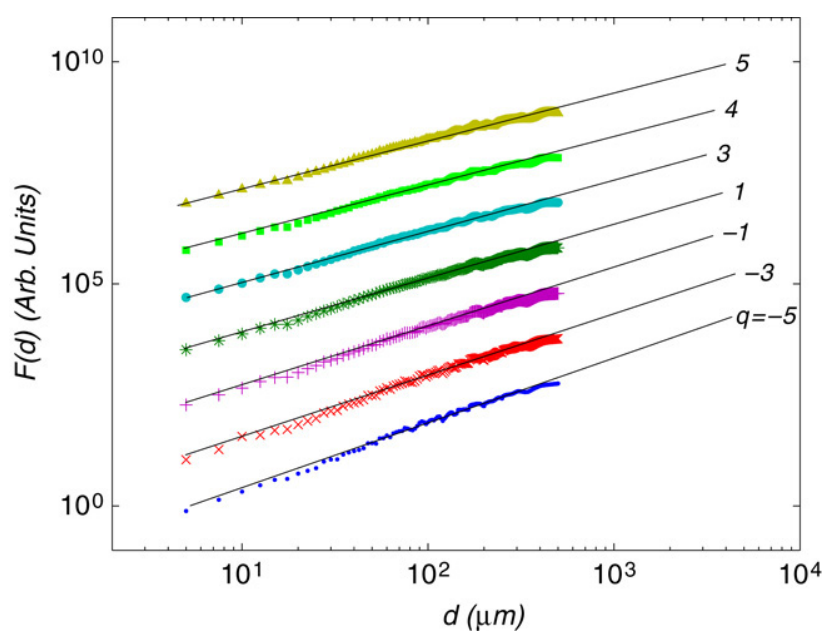

Fig. 2. $\log -\log$ plot of the fluctuation function $F_{q}(d)$ as a function of the distance $d=L \Delta x$, for a Py29 sample, for $q=-5,-3,-1,1,3,4$ and 5. The straight lines are the regression fits to the data. The slopes of the lines determine $h(q)$.

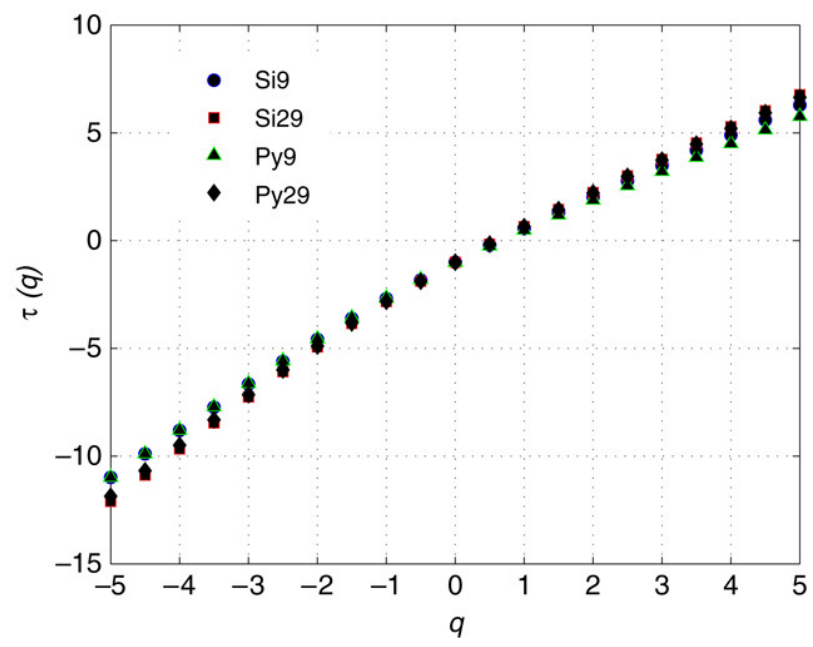

Fig. 3. The $q$ dependence of $\tau(q)=q h(q)-1$ for the four samples.

the roughness exponent of the profile. The strength of the multifractality of a profile is characterized by the difference between the maximum and minimum values of $\alpha$, i.e. $\Delta \alpha=\alpha_{\max }-\alpha_{\min }$.

Fig. 2 shows the $\log -\log$ plot of the fluctuation function $\left(F_{q}(d)\right)$ versus the distance $d=L \Delta x$, where $\Delta x$ is the spatial increment of the profile, for a Py29 sample. Also shown is the linear regression fit to the data for each value of $q$. We used a detrended polynomial of degree 1 (MFDFA1), but the result remained unchanged when polynomials of degree 2 and 3 were used (MFDFA2, MFDFA3). We can see clearly that the fluctuation functions $F_{q}(L)$ are straight lines in the double-logarithmic plot, with different slopes indicating the presence of multifractality. In Fig. 3 the plot of $\tau(q)$ versus $q$ is shown for the four samples Py9, Py29, Si9 and Si29. Note that for $|q|>5$, the function $\tau(q)$ coincides with its asymptotic form which is a linear function of $q .{ }^{1}$ Hence, we choose $-5 \leq q \leq 5$. We notice clearly that $\tau(q)$ is a concave function of $q$, typical of multifractal profiles. From Eq. (6) we can estimate the singularity spectrum for the four samples as shown in Fig. 4. This spectrum is averaged over 12 profile containing 8000 points each. In Table 2 we summarize the values of the strength of the multifractality $\Delta \alpha$ for the four samples alongside the value of $\alpha$ that maximizes the singularity spectrum.

\footnotetext{
${ }^{1}$ Note that the function $\tau(q)$ defined in the MFDFA formalism is equivalent to functions defined in standard multifractal formalism. For rigorous definitions and properties of $\tau(q)$ see for example [26].
} 


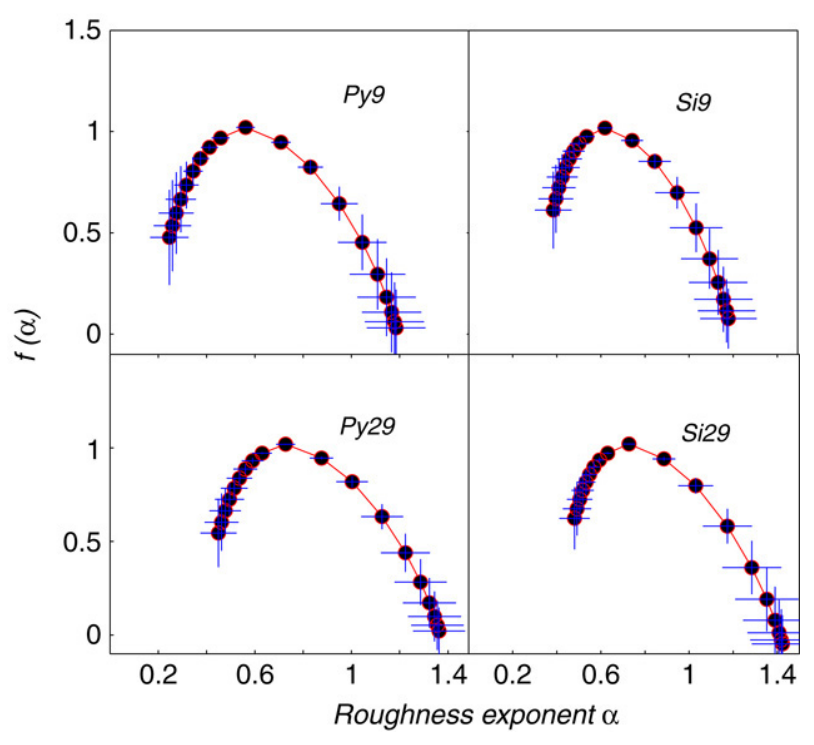

Fig. 4. The singularity spectrum for the four samples. In each case, the spectrum was averaged over 12 profiles containing 8000 points.

Table 2

Values of the multifractality strength $\Delta \alpha=\alpha_{\max }-\alpha_{\min }$ for different samples and the value of $\alpha$ that maximizes the multifractal spectrum

\begin{tabular}{lllll}
\hline Sample & Py9 & Si9 & Py29 & Si29 \\
\hline$\Delta \alpha$ & 0.94 & 0.8 & 0.91 & 0.94 \\
$\alpha_{\max }$ & 0.63 & 0.63 & 0.73 & 0.73 \\
\hline
\end{tabular}

\section{Discussion}

We showed that the surfaces generated by blasting particles share a common property of multifractality. The MFDFA method was used to uncover this property and to determine the singularity spectrum for each of the four samples. The strengths of multifractality represented by $\Delta \alpha$, as displayed in Table 2 , are close to each other regardless of the size of the particles and the material. The maximum of $f(\alpha)$ for Py29 and Si29 samples is attained for $\alpha_{\max } \simeq 0.73$, while for Py9 and Si9 samples this value is smaller, i.e. $\alpha_{\max } \simeq 0.63$ (Table 2). For samples Py 29 and $\mathrm{Si} 29$, the value of $\alpha_{\max }$ is very close to the universal value of 0.8 observed for fracture surfaces. Since the function $f(\alpha)$ can also be interpreted as the probability distribution of subsets characterized by $\alpha$ [20], $\alpha_{\max }$ corresponds to the dominant roughness exponent on the whole profile. Its value of 0.8 for Py 29 and Si29 samples suggests that it originates essentially from cracks formed by bombarding particles. This observation is in agreement with the accepted mechanism [7-9] which states that when hard particles hit a brittle material, the contact area is plastically deformed due to the high compressive and shear stresses and a radial crack is formed. After the impact, the plastic deformation leads to large tensile stresses that result in lateral cracks causing the material removal. In contrast, the value of $\alpha_{\max }$ for samples Py9 and $\mathrm{Si} 9$ is smaller than the universal value of 0.8 . For small particles ( $\mathrm{Si} 9$ and Py9 samples), the impacts are not powerful enough to initiate cracks and the erosion is ductile instead of brittle [27].

Similar multifractal behaviour was observed in the laser ablation of surfaces [28], which showed a pronounced multifractal spectrum. This spectrum and our spectrum demonstrate overlapping regions. However, no conclusion can be drawn regarding the similarities between the two erosion mechanisms as the laser ablation process is highly anisotropic, whilst the sharp particle bombardment is isotropic.

The observed multifractality on all samples can be interpreted in terms of spatial intermittency. This concept was argued by Krug [29] to describe the scaling of surfaces generated by epitaxial growth models incorporating very limited atomic mobility leading to a violent spatial intermittent effects and multifractal surfaces. This description was borrowed from fluid turbulence owing to the similarity between Galilean invariance of turbulent fluids and translational invariance of interfaces [30]. In our case, we highlight large fluctuations by considering the step size or the gradient at 


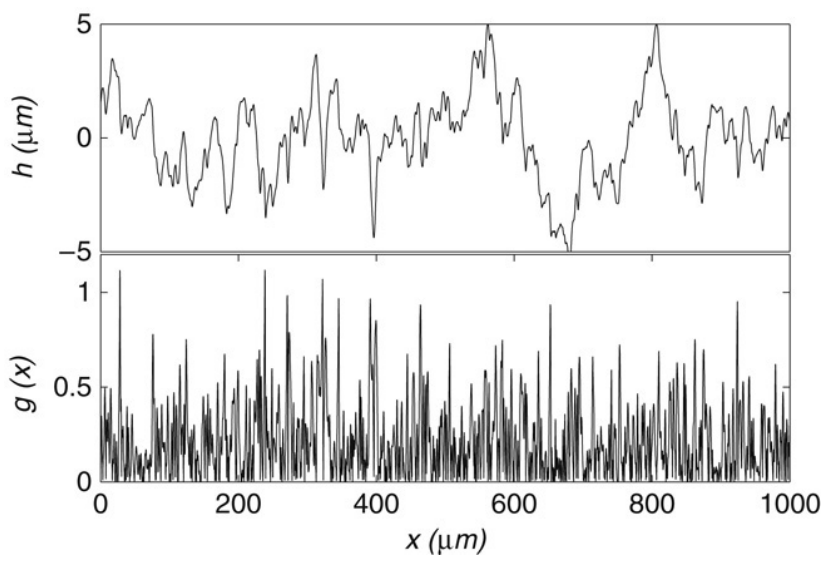

Fig. 5. Top plot, the surface profile of a Py29 sample with the corresponding local gradient (bottom plot) showing large fluctuations of the latter which constitutes the signature of intermittency.

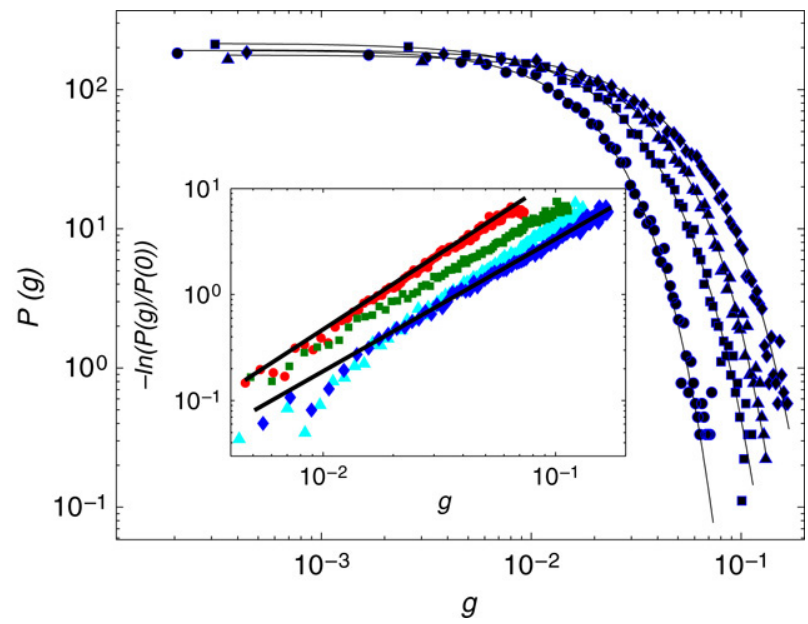

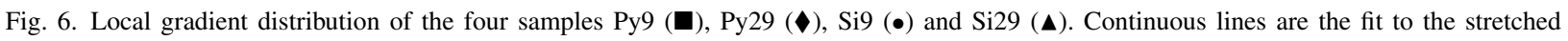
exponential distribution (7). The inset shows the $\log -\log$ plot of $-\ln (P(g) / P(0))$ as a function of $g$ where, the symbol designation is the same as in the main plot; the straight lines are shown to highlight the asymptotic power law scaling of $-\ln (P(g) / P(0))$ versus $g$.

the position $x, g(x)=|\partial h / \partial x|$ for a profile $h(x)$. This quantity is the analogue of the energy dissipation $\varepsilon=(\partial v / \partial x)^{2}$, where $v$ is the local velocity of a turbulent fluid [31].Fig. 5 shows the local gradient $g(x)$ for Pyrex glass bombarded with $29 \mu \mathrm{m}$ size particles, showing large gradient fluctuations, which suggests spatial intermittency. In addition, we consider the distribution of the local gradient in analogy with the local velocity gradient in fully developed turbulence, which is described by a stretched exponential distribution [32,33]. A very similar behaviour is characteristic of all four samples Py9, Py29, Si9 and Si29 as shown in Fig. 6, where the local gradient distribution fits very well with the stretched exponential function:

$$
P(g)=\frac{1}{\Omega} \exp \left(-a g^{\gamma}\right)
$$

The fit of the local gradient distribution to Eq. (7) gives the values of the stretching exponent $\gamma$, which are $1.41 \pm 0.05,1.23 \pm 0.03,1.37 \pm 0.05$ and $1.21 \pm 0.03$ for Si9, Py9, Si29 and Py29 respectively. The form of the local gradient distribution suggests that non-linearities must be present at large scale, leading to $h \rightarrow-h$ symmetry breaking. Indeed, the skewnesses $s$ defined as $s=(1 / \sqrt{N}) \sum_{i=1}^{N}\left(h_{i}-\bar{h}\right)^{3} / \sigma^{3}$ where $\bar{h}$ and $\sigma$ are the mean height and the standard deviation respectively, of the height distributions of the profiles of all samples are non-zero and have the values $s \simeq-0.4,-0.35,-0.28$ and -0.4 for Si9, Si29, Py9 and Py 29 respectively. We can now ask the question: 
What is the origin of the observed multifractality? It well known that multiplicative cascades [34,31] models generate processes known to have intermittent behaviour and a multifractal character, mirroring the presence of intermittent fluctuations with long range correlations. These long range correlations are generated at large scale by features that hierarchically cascade their influence to smaller scales. To detect the presence of long range correlations in our surface profiles, we perform the following test [35]: we generated a surrogate data set by shuffling the height data in each profile. The newly generated data set preserves the distribution of the height but destroys the long range correlations, which means that the surrogate profiles will exhibit a monofractal behaviour if the multifractality originates from the long range correlations and not from the height distribution. We performed the MFDFA and found that the surrogate data set is monofractal with the roughness exponent $\alpha=0.5$ for all samples. Thus, the observed multifractal behaviour is a result of long range correlations since the shuffling procedure preserves the height distribution. The effect of long range elastic interaction in developing long range correlations was reported by some authors in the case of elastic chains driven in a quenched random pinning [36], or during crack propagation [37]. However, a detailed theoretical investigation is needed in order to determine the origin of the long range correlations in sharp particle bombardment of brittle materials.

\section{Conclusion}

In conclusion, we conducted a detailed scaling analysis of the surfaces of two brittle materials, silicon and Pyrex glass, after bombardment with alumina particles of two different sizes $9 \mu \mathrm{m}$ and $29 \mu \mathrm{m}$. The bombardment results in multifractal surfaces. This multifractality is common to all samples regardless of the nature of the material or the size of the particles. We determined the corresponding singularity spectrum revealing a broad range of scaling exponents. We found that long range correlations are responsible for the observed multifractal behaviour. Although the full spectrum of roughness exponents can be fully determined by analysing one-dimensional cuts of surfaces, it is important to stress that a two-dimensional MFDFA (developed in Ref. [38]) is needed to fully characterize the multifractal behaviour of the blasted surfaces. Nevertheless, our characterization of one-dimensional cuts in different directions revealed this multifractal character and shows that long range correlations may play an important role in this blasting process.

\section{References}

[1] P. Slikkerveer, P. Bouten, F. de Haas, Sensors and Actuators 85 (2000) 296.

[2] S. Belloy, E. Thurre, A. Walckiers, Sayah, M. Gijs, Eurosensors XIII Proceeding, the Hague, Holland, 1999, p. 827.

[3] T.T. Veenstra, J.W. Berenschot, J.G.E. Gardeniers, R.G.P. Sanders, M.C. Elwenspoek, A. van den Berg, J. Electrochem. Soc. 148 (2001) G68.

[4] S. Schlautmann, H. Wensink, R.M. Schasfoort, M. Elwenspoek, A.V.D. Berg, J. Micromech. Microeng. 11 (2001) 386.

[5] P.J. Slikkerveer, P. Bouten, F. int Veld, H. Scholen, Wear 217 (1998) 237.

[6] X. Chen, J.W. Hutchinson, A.G. Evans, J. Amer. Ceram. Soc. 88 (2005) 1233.

[7] B.R. Lawn, E.R. Fuller, J. Mater. Sci. 10 (1975) 2016.

[8] D.B. Marshall, B.R. Lawn, A.G. Evans, J. Amer. Ceram. Soc. 65 (1982) 561.

[9] R.F. Cook, G.M. Pharr, J. Amer. Ceram. Soc. 73 (1990) 787.

[10] A.G. Evans, T.R. Wilshaw, Acta Mater. 24 (1976) 939.

[11] B.R. Lawn, J. Amer. Ceram. Soc. 81 (1998) 1977.

[12] A.G. Evans, E.A. Charles, J. Amer. Ceram. Soc. 59 (1976) 371.

[13] J.T. Hagan, M.V. Swain, J. Phys. D: Appl. Phys. 11 (1978) 2091.

[14] I. Finnie, Wear 186 (1995) 1.

[15] B. Lawn, Fracture of Brittle solids, Cambridge University Press, 1993.

[16] E. Bouchaud, J. Phys.: Condens. Matter 9 (1997) 4319.

[17] F. Family, T. Viscek, Dynamics of Fractal Surfaces, World Scientific, Singapore, 1991.

[18] K.J. Måløy, A. Hansen, E.L. Hinrichsen, S. Roux, Phys. Rev. Lett. 68 (1992) 213.

[19] J. Schmittbuhl, S. Roux, Y. Berthaud, Europhys. Lett. 28 (1994) 585.

[20] L. Calvet, A. Fisher, B.B. Mandelbrot, Cowles Foundation paper 1164 (1997).

[21] J.W. Kantelhardt, S.A. Zschiegner, E. Koscielny-Bunde, S. Havlin, A. Bunde, H.E. Stanley, Physica A 316 (2002) 87.

[22] J.F. Muzy, E. Bacry, A. Arneodo, Phys. Rev. Lett. 67 (1991) 3515.

[23] P. Oświęcimka, J. Kwapień, S. Drożdż, Phys. Rev. E 74 (2006) 016103.

[24] C.K. Peng, S.V. Buldyrev, S. Havlin, M. Simons, H.E. Stanley, A.L. Goldberger, Phys. Rev. E 49 (1994) 1685.

[25] J. Feder, Fractals, Plenum Press, New York, 1988.

[26] L. Calvet, A. Fisher, B.B. Mandelbrot, Cowles Foundation Discussion Paper No. 1165, 1997.

[27] H. Wensink, M.C. Elwenspoek, Wear 253 (2202) 1035. 
[28] K. Bube, C. Rodrigues Neto, R. Donner, U. Schwarz, U. Feudel, J. Phys. D: Appl. Phys. 39 (2006) 1405.

[29] J. Krug, Phys. Rev. Lett. 72 (1994) 2907.

[30] T. Bohr, G. Grinstein, C. Jayaprakash, M.H. Jensen, J. Krug, D. Mukamel, Physica A 59D (1992) 177.

[31] U. Frisch, Turbulence : The legacy of A. N. Kolmogorov, Cambridge University Press, 1995.

[32] A. Bershadskii, Europhys. Lett. 39 (1997) 587.

[33] P. Kailasnath, K.R. Sreenivasan, G. Stolovitzky, Phys. Rev. Lett. 68 (1992) 2766.

[34] B. Mandelbrot, On Intermittent Free Turbulence, Turbulence of Fluids and Plasmas, Interscience, New York, 1969.

[35] P.C. Ivanov, L.A.N. Amaral, A.L. Goldberger, S. Havlin, Michael, G. Rosenblum, Z.R. Struzikk, H.E. Stanley, Nature 399 (1999) 461.

[36] A. Tanguy, M. Gounelle, S. Roux, Phys. Rev. E 58 (1998) 1577.

[37] H. Gao, J.R. Rice, J. Appl. Mech. 56 (1989) 828.

[38] Gao-Feng Gu, Wei-Xing Zhou, Phys. Rev. E 74 (2006) 061104. 